\title{
Plans for a Proton Driver at Fermilab
}

\section{R. D. Kephart, Fermilab}

\section{Introduction}

During the last several years, stunning experimental results have established that neutrinos have nonzero masses and substantial mixing. The Standard Model must be extended to accommodate neutrino mass terms. The observation that neutrino masses and mass splittings are all many orders of magnitude smaller than those of any of the other fundamental fermions suggests radically new physics, perhaps originating at the GUT or Planck Scale, or perhaps the existence of new spatial dimensions. In some sense we know that the Standard Model is broken, but we don't know how it is broken. Whatever the origin of the observed neutrino masses and mixing, it is likely to require a profound extension to our picture of the physical world. The first steps in understanding this revolutionary new physics are to pin down the measurable parameters and to address the next round of basic questions:

- Are there only three neutrino flavors, or do light, sterile neutrinos exist?

- If there are only three generations, there is one angle $\left(\theta_{13}\right)$ in the mixing matrix that is unmeasured. How large is it?

- Which of the two possible orderings of the neutrino mass eigenstates applies?

- If $\theta_{13}$ is large enough one it may be possible to measure the quantummechanical phase $\delta$. If $\theta_{13}$ and $\delta$ are nonzero there will be $\mathrm{CP}$ violation in the lepton sector.

\footnotetext{
These questions can be addressed by accelerator based neutrino oscillation experiments. The answers will guide our understanding of what lies beyond the Standard Model, and whether the new physics provides an explanation for the baryon asymmetry of the Universe (via leptogenesis), or provides deep insight into the connection between quark and lepton properties (via Grand Unified Theories), or perhaps leads to an understanding of one of the most profound questions in physics: Why are there three generations of quarks and leptons? The answers may well further challenge our picture of the physical world, and will certainly have important implications for our understanding of
}

cosmology and the evolution of the early Universe.

The current Fermilab Program is an important part of the world-wide accelerator based effort to explore and understand the physics of neutrino oscillations. By early 2005, with both MINOS and MiniBooNE taking data, Fermilab will be able to answer some of the most pressing first-round questions raised by the discovery that neutrinos have mass. Fermilab's high-intensity neutrino beams are derived from 8- and $120-\mathrm{GeV}$ proton beams. MiniBooNE is currently taking data using $8 \mathrm{GeV}$ Protons from the Booster. The $120 \mathrm{GeV}$ NuMI beam will start to operate in early 2005 using a $0.25 \mathrm{MW}$ proton beam power from the Main Injector. Future neutrino programs will build on these existing facilities. New short and long baseline experiments have been proposed. There are proposals to increase the available number of protons at 8 and $120 \mathrm{GeV}$ with the goal of addressing the full range of questions presented by neutrino oscillations. Key to that vision is a new intense proton source that usually is referred to as the Proton Driver.

\section{Proton Driver}

Parts of Fermilab's existing Linac/Booster complex are nearly 35 years old. Maintenance and reliability are becoming a serious issue with these machines. Future new long baseline neutrino experiments will require further factors of 5-10 improvements in proton luminosity. It is clear that such experiments at Fermilab are only feasible if a major proton source upgrade is undertaken. The Proton Driver project would replace the Booster with a new $8-\mathrm{GeV}$ accelerator with $0.5-2 \mathrm{MW}$ beam power, a factor of $15-60$ more than the current Booster. It would also make the modifications needed to the Main Injector to upgrade it to simultaneously provide $120 \mathrm{GeV}$ beams of $2 \mathrm{MW}$.

A Proton Driver would bring with it other advantages. It would have the capacity to support a vigorous $8-\mathrm{GeV}$ fixed-target program while providing $2 \mathrm{MW}$ Main Injector beams. Several Fermilab reports have discussed the physics potential of such a program. A Proton Driver can also serve as a stepping-stone to future accelerators, both as an R\&D test bed and as an injector, with connections to the Linear Collider, Neutrino Factories, and a VLHC. 
Requirements for a new proton source can be met by either a new proton synchrotron or a superconducting linac. Main Injector RF upgrades would be common to both approaches as would beam handling and targeting systems.

\section{1 Synchrotron-based Proton Driver}

A design study for a $16-\mathrm{GeV}$ synchrotronbased Proton Driver was completed in December 2000 and documented in TM-2136. A second study, requested by the lab director for an $8-\mathrm{GeV}$ Proton Driver, was finished in May 2002 and documented in TM-2169, Part I. The main parameters of the second study (PD2) as well as that of the present Proton Source are listed in the following table. Compared to the existing Proton Source, the $8-\mathrm{GeV}$ synchrotron would increase the number of protons accelerated per cycle by a factor of five and the beam power at $8 \mathrm{GeV}$ by a factor of 15 .

\begin{tabular}{|c|c|c|}
\hline Parameters & $\begin{array}{l}\text { Present } \\
\text { Proton } \\
\text { Source }\end{array}$ & $\begin{array}{l}\text { Proton } \\
\text { Driver (PD2) }\end{array}$ \\
\hline \multicolumn{3}{|l|}{$\begin{array}{l}\text { Linac (operating } \\
\text { at } 15 \mathrm{~Hz} \text { ) }\end{array}$} \\
\hline $\begin{array}{ll}\text { Kinetic energy } \\
(\mathrm{MeV})\end{array}$ & 400 & 600 \\
\hline $\begin{array}{ll}\begin{array}{l}\text { Peak } \\
(\mathrm{mA})\end{array} & \text { current } \\
\end{array}$ & 40 & 50 \\
\hline Pulse length $(\mu \mathrm{s})$ & 25 & 90 \\
\hline \multicolumn{3}{|l|}{$\begin{array}{l}\text { Booster } \\
\text { (operating at } 15 \\
\mathrm{~Hz} \text { ) }\end{array}$} \\
\hline $\begin{array}{l}\text { Extraction kinetic } \\
\text { energy }(\mathrm{GeV})\end{array}$ & 8 & 8 \\
\hline Protons per cycle & $5 \times 10^{12}$ & $2.5 \times 10^{13}$ \\
\hline Protons per hour & $9 \times 10^{16 *}$ & $1.35 \times 10^{18}$ \\
\hline $\begin{array}{l}\text { Beam power } \\
(\mathrm{MW})\end{array}$ & $0.033(*)$ & 0.5 \\
\hline
\end{tabular}

(*) Continuous operation at $5 \mathrm{~Hz}$

There are several drawbacks to the upgrade approach described in TM-2169. Portions of the existing 35 year old linac would remain with associated maintenance issues. The required large aperture rapid cycling magnets would be expensive to develop and build. The proposal as currently envisioned requires extensive interference with the current Run II and planned $\mathrm{BTeV}$ physics programs for civil construction and equipment moves. Five booster cycles are required to fill the main injector. This takes $1 / 3$ of a second out of the timeline limiting the maximum repetition rate and thus the delivered beam power. Finally, instabilities and resultant emittance blowup in the new booster might lead to beam losses in either the new booster or main injector that would limit the ultimate performance of such a machine to less than the stated goals.

\section{Superconducting Linac}

A design study for a Superconducting Linacbased Proton Driver has been completed and is being documented in Fermilab-TM-2169, Part II. The table below shows the principle parameters of such a machine.

The SC linac would accelerate an $\mathrm{H}$ beam that is subsequently injected and stripped in the Main Injector. This avoids the space-charge tune spread in a booster synchrotron. The simplicity of design should make it simpler to operate than booster/linac combinations. Limited emittance growth in a linac means that it can deliver the high brightness, low halo beams needed for running the Main Injector at high intensity with acceptable losses. The short MI "fill time" $(<1$ ms) for the SCRF linac means that the MI could deliver the full $2 \mathrm{MW}$ of beam power at any energy from 40 to $120 \mathrm{GeV}$, and that improvements to the MI ramp time could further increase the average proton intensity. The $8 \mathrm{GeV}$ superconducting linac could also accelerate electrons. There are many technical overlaps between the development and construction of such a machine and a cold technology Linear Collider.

A major question for the $8-\mathrm{GeV}$ Linac is cost. In a linac, the expensive radio frequency (RF) systems must transfer their energy to the beam in a single pass, whereas in a synchrotron the RF costs are amortized over many thousands of beam passages through the accelerating cavities. As a result, synchrotrons have traditionally been preferred for attaining the highest beam energies, while linacs (due to their simplicity and relative immunity to space charge effects) have traditionally been the preferred solution for the low energy (few hundred $\mathrm{MeV}$ ) injector to such machines. Recent developments have dramatically reduced the costper-GeV of superconducting linacs, and have the potential to move the optimal technological crossover point substantially upwards in energy. Currently we envision a design based to the extent possible on the SCRF technology developed for TESLA. Figure 1 shows the baseline SCRF Proton Driver. This version utilizes an RFQ, a warm Drift Tube Linac, $1300 \mathrm{Mhz}$ "squeezed" Tesla cavities for the beta $<1$ portion of the linac, and modified beta $=1$ TESLA cryomodules above $1.2 \mathrm{GeV}$. The baseline design could accelerate 2 MW of beam at $8 \mathrm{GeV}$ while simulataneously delivering $2 \mathrm{MW}$ of beam at $120 \mathrm{GeV}$ from the Main Injector. We are investigating various staging options that could reduce the klystron 


\begin{tabular}{|l|l|}
\hline PRIMARY PARAMETERS & \multicolumn{1}{|c|}{$\mathbf{8 ~ G e V ~ L i n a c ~}$} \\
\hline Linac Particle Type & $\mathrm{H}^{-}$ions or Electrons selectable on pulse-by-pulse \\
\hline Linac beam kinetic energy & $8 \mathrm{GeV}$ \\
\hline Linac Beam power & $2 \mathrm{MW}$ sum of $\mathrm{H}$ - and e- at $8 \mathrm{GeV}$ \\
\hline Linac Pulse repetition rate & $10 \mathrm{~Hz}$ combined for $\mathrm{H}$ - and e- \\
\hline Linac macropulse width & $1 \mathrm{~ms}$ \\
\hline Linac current (avg. in macropulse) & $26 \mathrm{~mA}$ \\
\hline Linac Particles per macropulse & $1.56 \mathrm{E}+14 \mathrm{H}$ - or e- \\
\hline Linac beam macropulse duty factor & $1 \%$ \\
\hline Linac RF duty factor & $1.3 \%$ \\
\hline Linac Active Length & $692 \mathrm{~m}$ \\
\hline Ring circumference & $3319.4 \mathrm{~m}$ Fermilab Main Injector \\
\hline Ring Beam Energy & $8-120 \mathrm{GeV}$ \\
\hline Ring Beam Power on Target & $2 \mathrm{MW} \sim$ independent of MI Beam Energy \\
\hline Ring Circulating Current & $2.3 \mathrm{~A}$ \\
\hline Ring cycle time & $0.2-1.5$ sec depends on MI beam energy \\
\hline Ring Protons per Pulse on Target & $1.5 \mathrm{E}+14$ protons \\
\hline Ring Proton pulse length on Target & $10 \mathrm{us} 1$ turn, or longer with resonant extraction \\
\hline Wall Power at 10Hz Operation & $12 \mathrm{MW}$ approx $2 \mathrm{MW}$ Standby $+1 \mathrm{MW} / \mathrm{Hz}$ \\
\hline
\end{tabular}

count in order to reduce the initial cost at the expense of $8 \mathrm{GeV}$ power. We are also exploring options that replace the copper DTL with Superconducting spoke resonators that have been developed at MSU and Argonne National Laboratory for the RIA project. Figure 2 shows a possible staged version of the machine with a superconducting front-end, and reduced klystron and modulator count. Such a machine would still deliver $2 \mathrm{MW}$ from the Main Injector but only 0.5 MW at $8 \mathrm{GeV}$. However, the savings in initial cost of such a staged machine are expected to be substantial.

\section{Changes needed in the Main Injector}

The Main Injector (MI) currently is designed to operate at 3.0E13 protons per pulse (ppp) at $120 \mathrm{GeV} / \mathrm{c}$ or $150 \mathrm{GeV} / \mathrm{c}$. Proton beam intensities with a Proton Driver will be considerably larger than the MI original design capability. Systematic upgrades to the MI complex are required prior to a Proton Driver to meet the demands of the anticipated FNAL physics program and to prepare the MI for a future high intensity Proton Driver.

Considerable increase in the integrated proton flux is possible by reducing the Main Injector cycle time. The fill time of the Main Injector is at present limited by the Booster which cycles at 15 Hz. A linac based Proton Driver would remove this limitation. A preliminary study shows that with an 8-Gev linac the MI cycle time can be reduced from $1.9 \mathrm{sec}$ to $1.0 \mathrm{sec}$ or less by a modest investment in the MI power supplies and RF cavities. Doubling the maximum available power supply voltage can increase the magnet ramp rate. This can be achieved by adding two dipole power supplies and one quadrupole power supply to every MI Service Building. This faster ramp rate and increased flux will require substantial increases in the RF power in the Main Injector. The current Main Injector RF cavities can handle $6 \mathrm{E} 13 \mathrm{ppp}$ vs the planned 1.5E14 ppp from a Proton Driver. Two possible paths for the cavity upgrade are envisioned 1) increase the number of RF cavities or 2) design and build new cavities that can handle the higher flux and ramp rate. $R \& D$ will be required to determine the optimal Main Injector RF cavity upgrade.

Higher MI bunch intensity will require additional upgrades including: 1) improvements to the damper system to reduce instabilities driven by higher intensities. 2) A Gamma-t system to control the growth of longitudinal emittance through transition. 3) Aperture improvements. 4) Improved Radiation Shielding and collimation. 5) $\mathrm{H}$ - Injection (in the case of the SCRF Linac). 6) Target upgrades to handle MW class beams.

\section{Plans and Acknowledgements}

Our near term plans are to develop these ideas and perform critical $R \& D$ over the next year with the goal of submitting a proposal in 2005 to the US Department of Energy to construct a new intense proton source at Fermilab. The author acknowledges that this work is the product of the effort of many individuals and refers the reader to the referenced technical documents for names of other contributors. 


\section{Baseline 2 MW 8 GeV LINAC}

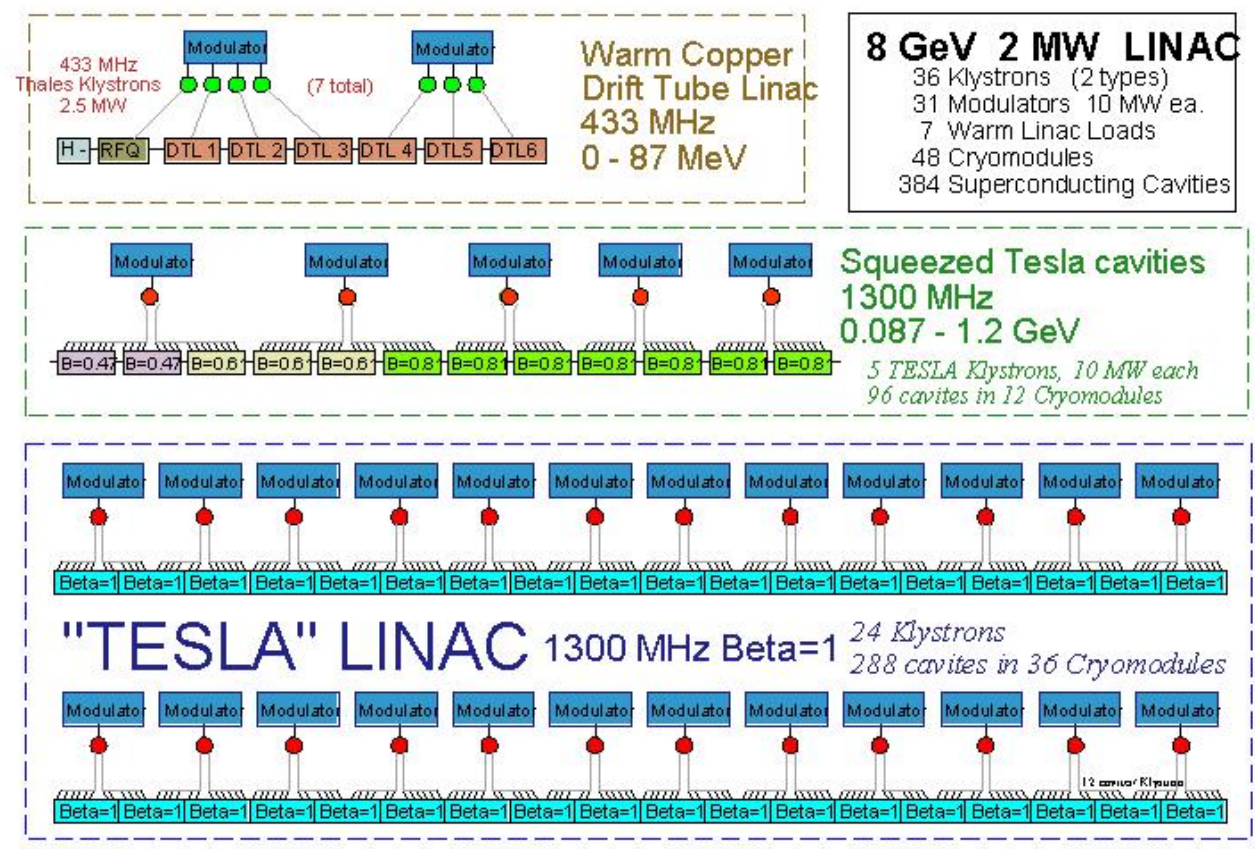

Figure 1: Baseline SCRF linac based Proton Driver. This version employs an RFQ and a warm copper Drift Tube Linac as the front end. It can deliver $2 \mathrm{MW}$ of beam at 120 $\mathrm{GeV}$ and simultaneously $2 \mathrm{MW}$ of beam at $8 \mathrm{GeV}$.

\section{Staged:2MW@120GeV \&.5 MW@8GeV,SCRFFE}

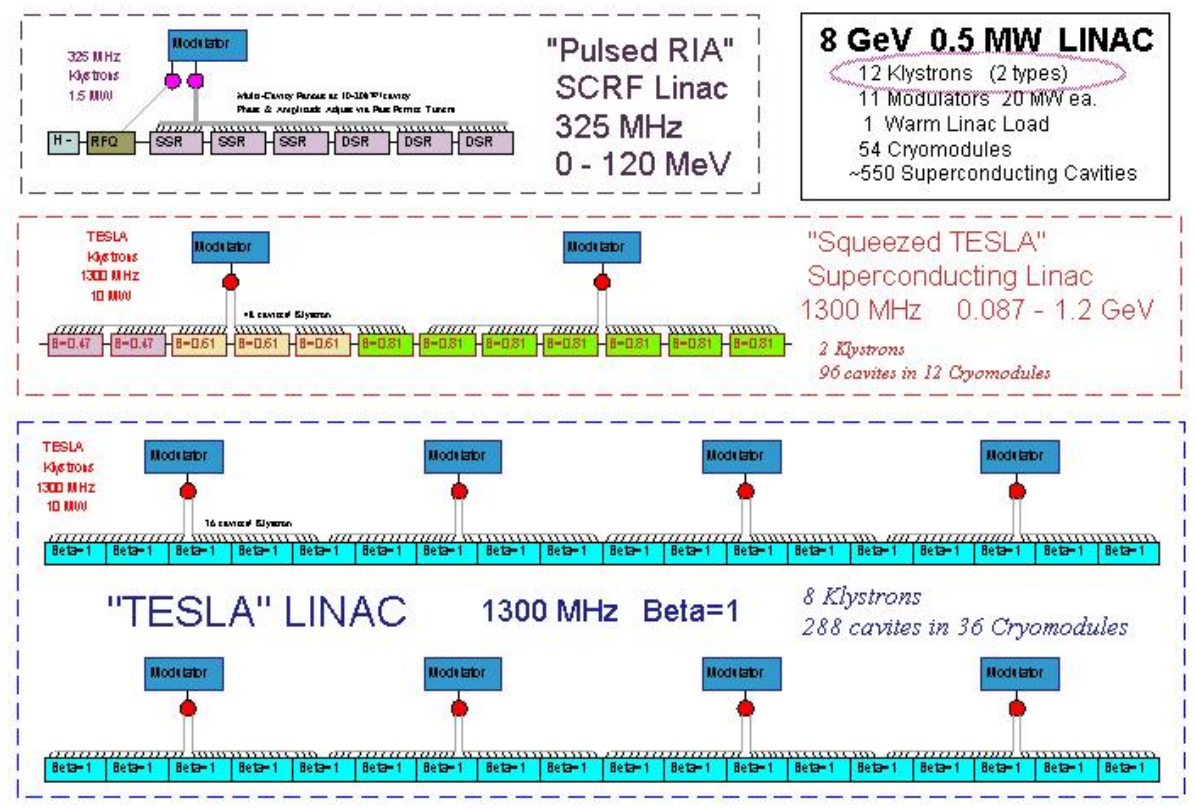

Figure 2: Possible staging option for SCRF linac based Proton Driver. This version employs an RFQ and a superconducting the front end. It can deliver $2 \mathrm{MW}$ of beam at $120 \mathrm{GeV}$ and simultaneously $0.5 \mathrm{MW}$ of beam at $8 \mathrm{GeV}$. 